\title{
Combination of Pembrolizumab With Platinum-containing Chemotherapy for Pleomorphic Carcinoma of the Lung
}

\author{
SHINICHIRO OKAUCHI ${ }^{1}$, YUIKA SASATANI ${ }^{1}$, TOSHIHIRO SHIOZAWA ${ }^{2}$, \\ HIDEYASU YAMADA ${ }^{3}$, KUNIHIKO MIYAZAKI ${ }^{4}$, NORIO TAKAYASHIKI ${ }^{5}$ and HIROAKI SATOH ${ }^{1}$ \\ ${ }^{1}$ Division of Respiratory Medicine, Mito Medical Center, \\ University of Tsukuba-Mito Kyodo General Hospital, Mito, Japan; \\ ${ }^{2}$ Division of Respiratory Medicine, Faculty of Medicine, University of Tsukuba, Tsukuba, Japan; \\ ${ }^{3}$ Division of Respiratory Medicine, Hitachinaka Medical Center, \\ University of Tsukuba-Hitachinaka General Hospital, Hitachinaka, Japan; \\ ${ }^{4}$ Division of Respiratory Medicine, Ryugasaki Saiseikai General Hospital, Ryugasaki, Japan; \\ ${ }^{5}$ Division of Pathology, Mito Medical Center, University of Tsukuba-Mito Kyodo General Hospital, Mito, Japan
}

\begin{abstract}
Background/Aim: Pleomorphic carcinoma of the lung is a rare, highly malignant subtype of lung cancer, with a more aggressive clinical course compared with other types of non-small-cell lung cancer (NSCLC). Platinum-containing chemotherapy has been the standard therapy for patients with NSCLC and pembrolizumab is one of the novel and reliable agents for these patients. Case Report: We herein report the case of a 60-year-old man with advanced chemo-naive pleomorphic carcinoma of the lung who was successfully treated with a combination of pembrolizumab with platinumcontaining chemotherapy. Conclusion: In the absence of definitive clinical trials, which are unlikely to be performed due to the rarity of this tumor, our case demonstrates the potential utility of the combination of pembrolizumab with platinum-containing chemotherapy. Our result also suggest that this combination of therapy may be key to the treatment of pleomorphic carcinoma of the lung.
\end{abstract}

Pleomorphic carcinoma of the lung is a rare, highly malignant tumor, accounting for $0.1-0.4 \%$ of all primary lung cancer cases (1-3). It has a more aggressive clinical course compared with other types of non-small-cell lung cancer (NSCLC) (4-6), as well as a poorer outcome $(4,7,8)$.

This article is freely accessible online.

Correspondence to: Hiroaki Satoh, MD, Ph.D., Division of Respiratory Medicine, Mito Medical Center, University of TsukubaMito Kyodo General Hospital, Miya-machi 3-2-7, Mito-city, Ibaraki, 310-0015, Japan. E-mail: hirosato@md.tsukuba.ac.jp

Key Words: Combination chemotherapy, immune checkpoint inhibitor, pembrolizumab, pleomorphic carcinoma, lung.
Immune checkpoint inhibitors have significantly changed the treatment of patients with advanced NSCLC. The time has come for long-term survivors and cured patients to emerge. However, the fact that there are patients who do not respond at all to immune checkpoint inhibitors cannot be ignored. Therefore, there is a trend for improving treatment results by using immune checkpoint inhibitors in combination with chemotherapeutic drugs. Recently, there have been some case reports of patients with polymorphic cancer responding to immune checkpoint inhibitors (9-13), while others have failed (9-13). We herein report a case of pleomorphic carcinoma of the lung treated with pembrolizumab and platinum-containing chemotherapy. Our results demonstrate that this combination chemotherapy may be key to the treatment of advanced cases with this type of lung cancer.

\section{Case Report}

A 60-year-old man was referred to our hospital with complaints of chest and occipital discomfort, and general fatigue. He was an architect and had no smoking habit. Chest radiograph and computed tomographic (CT) scan revealed a tumor of $3 \mathrm{~cm}$ in diameter in the left lung with enlargement of ipsilateral mediastinal lymph nodes (Figures 1 and 2). Biopsy specimens, which were obtained from the primary lesion and from a rib metastasis, were diagnosed as NSCLC. Chest and abdominal CT scan, brain magnetic resonance imaging and bone scan showed distant metastasis to the $8^{\text {th }}$ right rib and left adrenal gland. Clinical stage was T3N3M1c stage IVB. During these diagnostic procedures, the patient's performance status rapidly deteriorated. We selected chemotherapy containing pembrolizumab (200 mg, q28), carboplatin (area under the curve of $=5, q 28$ ), and paclitaxel $\left(180 \mathrm{mg} / \mathrm{m}^{2}, q 28\right)$. After the third cycle of the chemo- 


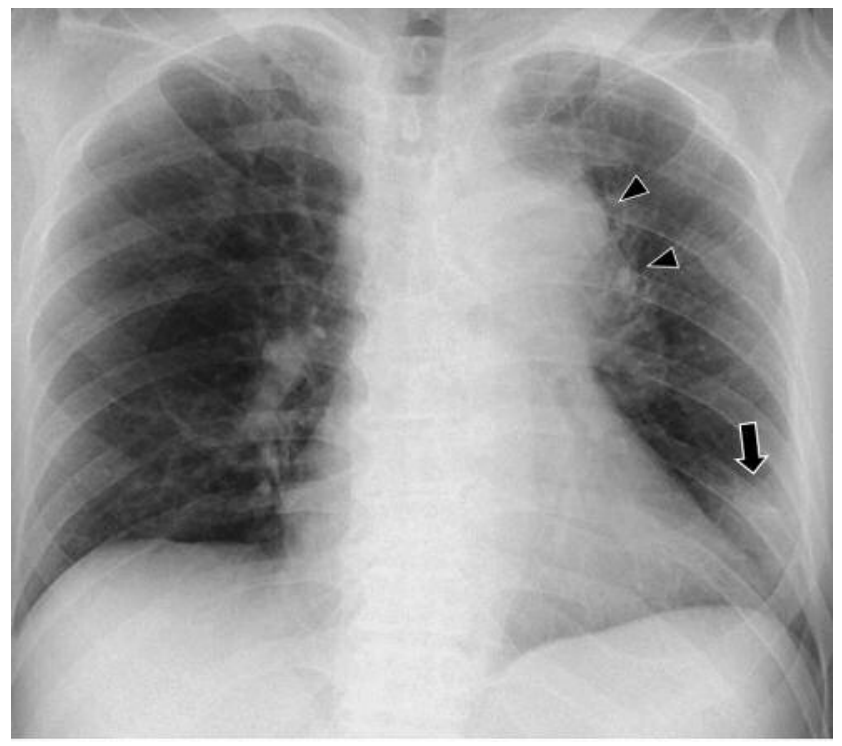

Figure 1. Chest radiograph showed a tumor $3 \mathrm{~cm}$ in diameter in the left lung (arrow) with enlargement of ipsilateral mediastinal lymph nodes (arrowheads).

combination therapy, the primary lesion in left lung and ipsilateral mediastinal lymph nodes were found to have shrunk on chest radiograph and CT (Figures 3 and 4). The patient's general condition rapidly improved. There were no severe adverse effects of more than grade II anorexia. Six cycles of this chemo-combination therapy were performed. After the initiation of the treatment, the following detailed pathological diagnosis results were obtained. Tumor consisted of undifferentiated polymorphic malignant cells. The tumor cells were highly atypical, with distinct nucleoli, heterogeneous or bizarre megakaryocytes and polynuclei. These tumor cells grew solid without forming a specific structure. Immunostaining of tumor cells was positive for cytokeratin 7 (CK7), but negative for thyroid transcription factor 1, p40, S-100, and CK20. There was neither epidermal growth factor receptor/c-ros oncogene 1 gene mutation nor anaplastic lymphoma kinase fusion gene. However, programmed death ligand 1 (PD-L1) tumor proportion score was over $75 \%$. The final pathological diagnosis was pleomorphic carcinoma of the lung. The patient remains well, 7 months after the diagnosis, and will be receiving maintenance therapy with pembrolizumab.

\section{Discussion}

Pleomorphic carcinomas are infrequent, comprising 0.1-0.4\% of all lung tumors (1-3). Several studies have reported that pleomorphic carcinomas are associated with a more advanced stage at presentation and a poorer prognosis $(1,5$,
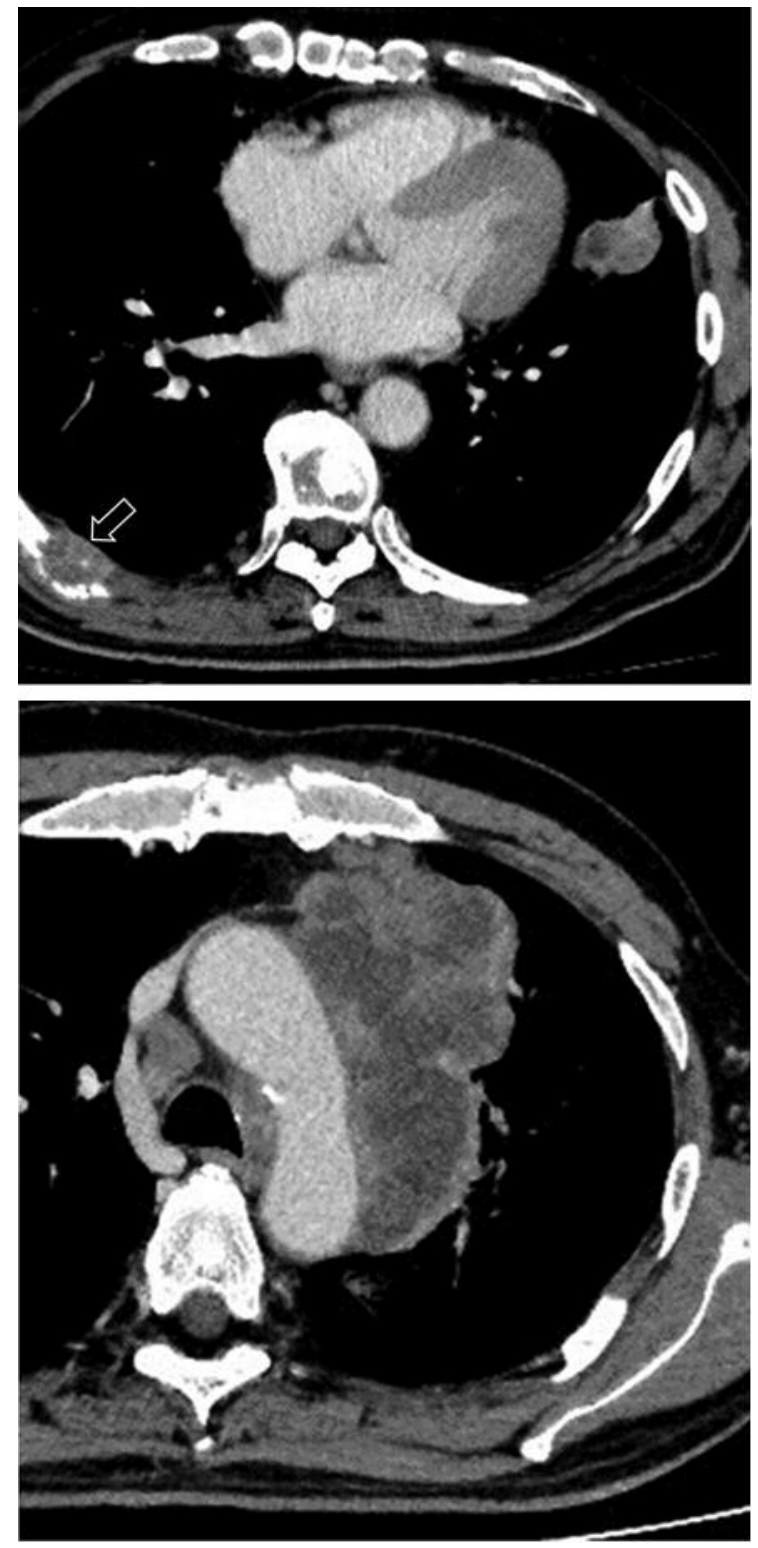

Figure 2. Chest computed tomographic scan revealed a tumor $3 \mathrm{~cm}$ in diameter in the left lung with enlargement of ipsilateral mediastinal lymph nodes, and an osteolytic mass of the $8^{\text {th }}$ right rib due to metastasis (arrow).

$8,14)$. The average age of patients with pleomorphic carcinoma is 60 to 65 years, the male-female ratio is $5: 1$, and 60 to $90 \%$ of patients with this type of NSCLC have a smoking habit (15). It was recently reported that the expression of PD-L1 in this type of NSCLC is relatively high $(9,13,16)$. Some patients with pleomorphic carcinoma with a smoking habit and high PD-L1 expression may benefit from immune checkpoint inhibitors. Immune checkpoint inhibitors such as antibodies to programmed death 1 

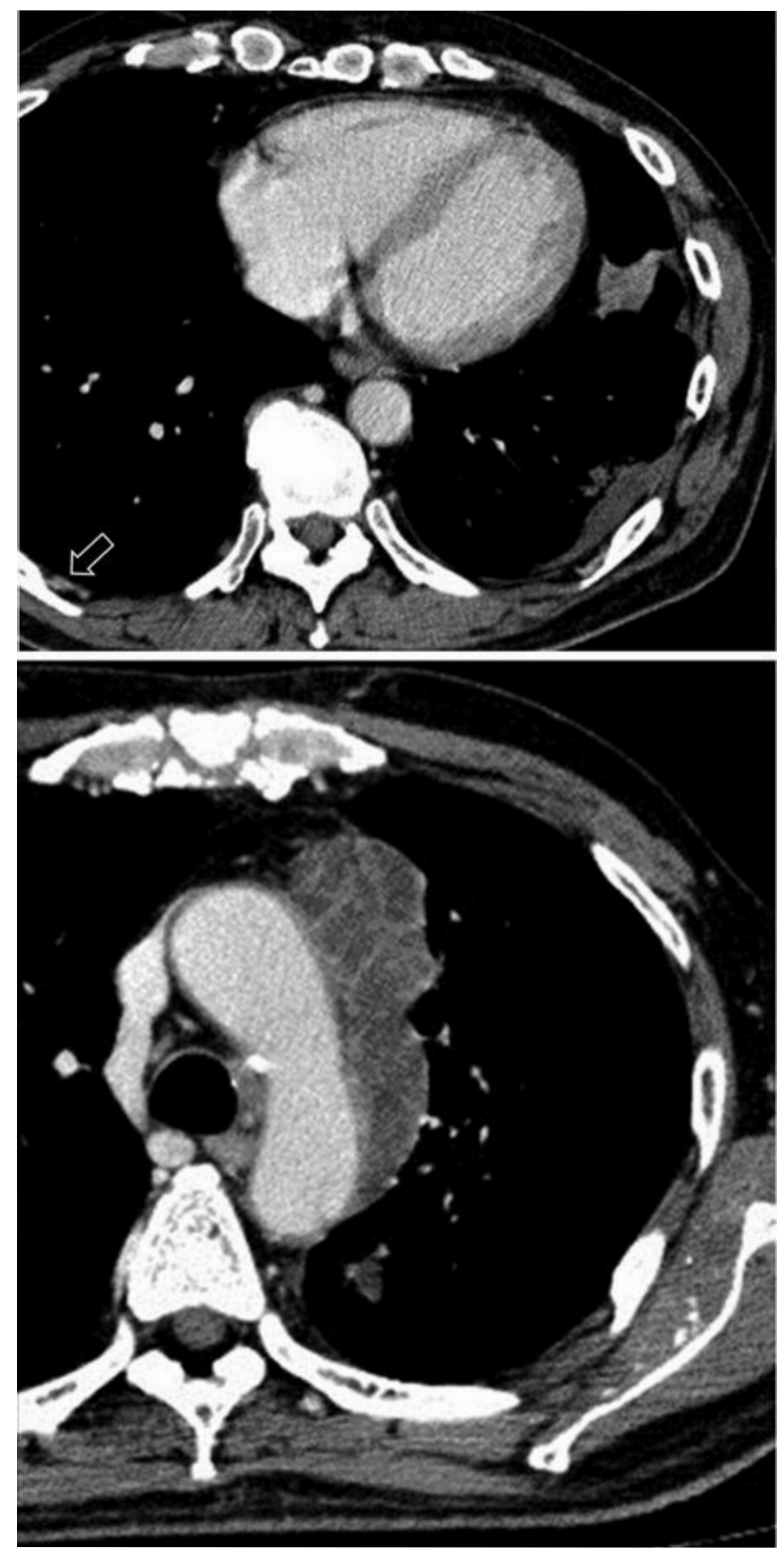

Figure 3. Chest computed tomographic scan taken after the third cycle of the chemo-combination therapy revealed shrinkage of the primary lesion and mediastinal lymph nodes. Shrinkage of the metastatic mass of the $5^{\text {th }}$ right rib was also apparent (arrow).

(PD1)/PD-L1 have dramatically changed the treatment paradigm for NSCLC (3). Studies have also shown associations between PD-L1 expression, smoking and tumor mutational load as potential determinants of response to PD1/PD-L1 inhibitors (4). There have been some recent reports of cases successfully treated with immune checkpoint inhibitors such as nivolumab and pembrolizumab (9-13), although patients who did not respond have also been reported (13). Because of rarity of this type of NSCLC, it is

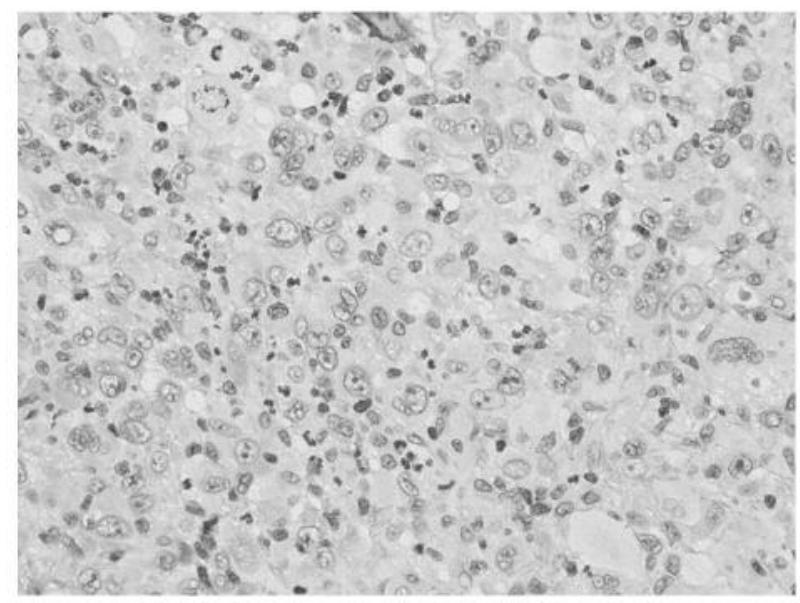

Figure 4. Pathological specimen from the primary lesion. The tumor consisted of undifferentiated polymorphic malignant cells. The tumor cells were highly atypical, with distinct nucleoli, heterogeneous or bizarre megakaryocytes and polynuclei. These tumor cells grew solid without forming a specific structure (hematoxylin-eosin staining, $\times 400$ ).

difficult to clarify the effect of immune checkpoint inhibitors on this type of NSCLC in clinical trials. Given the outcomes of these responders, however, immune checkpoint inhibitors may be considered as one of the treatment options for patients with pleomorphic carcinoma, especially those with a high PD-L1 level.

In survival curves for patients those with immune checkpoint inhibitor for NSCLC, a 'tail plateau' has been noted. For any immune checkpoint inhibitor, however, there were patients that experienced recurrence after a period of some effect and patients that experienced no survival benefit. Combinations of immune checkpoint inhibitors and traditional antitumor agents have been studied in clinical trials to improve the prognosis of patients in these groups (17, 18). A particularly noteworthy trial was KEYNOTE-021, which showed clinical superiority of pembrolizumab and platinum-based chemotherapy as first-line therapy for advanced NSCLC (17). Prior to the advent of immune checkpoint inhibitors, paclitaxel was administered to patients with pleomorphic carcinoma, and there have been some case reports of good response without serious adverse effects (1820). In the present patient, the provisional pathological diagnosis at the time of obtaining pathological specimen tissue was non-squamous NSCLC. Because the disease progressed, treatment with pembrolizumab and platinumcontaining chemotherapy was initiated. Thereafter, pathological examination confirmed that the patient had pleomorphic carcinoma of the lung. In the chest CT performed after the first course of the therapy, as there was shrinkage of the primary lesion, this therapy has been continued until now. This patient had no smoking habit but 
cancer tissue obtained from the primary tumor had high expression of PD-L1. Such a patient background is advantageous for a response to treatment with pembrolizumab and platinum-containing chemotherapy.

As described above, there have been some case reports of treatment with immune checkpoint inhibitors for pleomorphic carcinoma. However, to our knowledge, no report of patients responding to pembrolizumab and platinum-containing chemotherapy for pleomorphic carcinoma was found. The duration of response for such treatments is of great interest. The advantages and disadvantages of using immune checkpoint inhibitors alone and in combination with chemotherapy are also interesting. It is meaningful to accumulate clinical experience in order to create better treatments for this rare type of carcinoma. Our case demonstrates the potential utility of pembrolizumab and platinum-containing chemotherapy.

\section{Statement of Ethics}

This study was approved by the institutional Ethics Committee of each Hospital (Project approval number: NO16-66). Written comprehensive informed consent at the time of admission for obtaining pathological specimens was obtained from the patient.

\section{Conflicts of Interest}

None to be declared.

\section{Authors' Contributions}

SH and HS designed the study. SO, YS, TS, HY and KM collected the data. SH, KM, NT and HS analyzed the data and prepared the article. All Authors approved the final version of the article.

\section{References}

1 Chang YL, Lee YC, Shih JY and Wu CT: Pulmonary pleomorphic (spindle) cell carcinoma: Peculiar clinicopathologic manifestations different from ordinary non-small cell carcinoma. Lung Cancer 34(1): 91-97, 2001. PMID: 11557118. DOI: 10.1016/s0169-5002(01)00224-0

2 Ito K, Oizumi S, Fukumoto S, Harada M, Ishida T, Fujita Y, Harada T, Kojima T, Yokouchi H and Nishimura M; Hokkaido Lung Cancer Clinical Study Group: Clinical characteristics of pleomorphic carcinoma of the lung. Lung Cancer 68(2): 204210, 2010. PMID: 19577320. DOI: 10.1016/j.lungcan. 2009.06.002

3 Pelosi G, Sonzogni A, De Pas T, Galetta D, Veronesi G, Spaggiari L, Manzotti M, Fumagalli C, Bresaola E, Nappi O, Viale $\mathrm{G}$ and Rosai J: Review article: Pulmonary sarcomatoid carcinomas: A practical overview. Int J Surg Pathol 18(2): 103120, 2010. PMID: 19124452. DOI: $10.1177 / 1066896908330049$

4 Mochizuki T, Ishii G, Nagai K, Yoshida J, Nishimura M, Mizuno T, Yokose T, Suzuki K and Ochiai A: Pleomorphic carcinoma of the lung: Clinicopathological characteristics of 70 cases. Am J
Surg Pathol 32(11): 1727-1735, 2008. PMID: 18769330. DOI: 10.1097/PAS.0b013e3181804302

5 Fishback NF, Travis WD, Moran CA, Guinee DG Jr., McCarthy WF and Koss MN: Pleomorphic (spindle/giant cell) carcinoma of the lung. A clinicopathologic correlation of 78 cases. Cancer 73(12): 2936-2945, 1994. PMID: 8199991. DOI: 10.1002/10970142(19940615)73:12<2936::aid-cncr2820731210>3.0.co;2-u

6 Rossi G, Cavazza A, Sturm N, Migaldi M, Facciolongo N, Longo L, Maiorana A and Brambilla E: Pulmonary carcinomas with pleomorphic, sarcomatoid, or sarcomatous elements: A clinicopathlogic and immunohistochemical study of 75 cases. Am J Surg Pathol 27(3): 311-324, 2003. PMID: 12604887. DOI: 10.1097/00000478-200303000-00004

7 Yamamoto S, Hamatake D, Ueno T, Higuchi T, Hiratsuka M, Shiraishi T, Iwasaki A and Shirakusa T: Clinicopathological investigation of pulmonary pleomorphic carcinoma. Eur J Cardiothorac Surg 32(6): 873-876, 2007. PMID: 17942316. DOI: $10.1016 /$ j.ejcts.2007.09.010

8 Bae HM, Min HS, Lee SH, Kim DW, Chung DH, Lee JS, Kim YW and Heo DS: Palliative chemotherapy for pulmonary pleomorphic carcinoma. Lung Cancer 58(1): 112-115, 2007. PMID: 17574296. DOI: 10.1016/j.lungcan.2007.05.006

9 Okamura K, Fukuda Y, Soda H, Ogawara D, Iwasaki K, Fuchi S, Suyama T, Yoshida M, Harada T, Fukuda M and Mukae H: Pulmonary pleomorphic carcinoma with few PD-1-positive immune cells and regulatory T-cells that showed a complete response to nivolumab. Thorac Cancer 9(1): 193-196, 2018. PMID: 29131510. DOI: 10.1111/1759-7714.12557

10 Ito K, Hataji O, Katsuta K, Kobayashi T, Gabazza EC, Yatabe Y, Taguchi $\mathrm{O}$ and Yamamoto N: 'Pseudoprogression' of pulmonary pleomorphic carcinoma during nivolumab therapy. J Thorac Oncol 11(10): e117-e119, 2016. PMID: 27189927. DOI: 10.1016/ j.jtho.2016.05.002

11 Ikematsu Y, Yoneshima Y, Ijichi K, Tanaka K, Harada T, Oda Y, Nakanishi Y and Okamoto I: Marked response to pembrolizumab in a patient with pulmonary pleomorphic carcinoma highly positive for PD-L1. Lung Cancer 112: 230-231, 2017. PMID: 28754417. DOI: 10.1016/j.lungcan.2017.07.020

12 Matsumoto Y, Miura T, Horiuchi H and Usui K: The successful treatment of pulmonary pleomorphic carcinoma with pembrolizumab: A case report. Case Rep Oncol 10(2): 752-757, 2017. PMID: 28878661. DOI: 10.1159/000479552

13 Kanazu M, Uenami T, Yano Y, Nakatsubo S, Hosono Y, Ishijima M, Akazawa Y, Yamaguchi T, Urasaki K, Mori M and Yokota S: Case series of pleomorphic carcinomas of the lung treated with nivolumab. Thorac Cancer 8(6): 724-728, 2017. PMID: 28881488. DOI: $10.1111 / 1759-7714.12505$

14 Raveglia F, Mezzetti M, Panigalli T, Furia S, Giuliani L, Conforti $S$ and Meda $S$ : Personal experience in surgical management of pulmonary pleomorphic carcinoma. Ann Thorac Surg 78(5): 1742-1747, 2004. PMID: 15511465. DOI: 10.1016/ j.athoracsur.2004.04.084

15 Kaira K, Shimizu K, Endoh H, Imaizumi K, Kamiyoshihara M, Sugano M, Kawashima O, Tanaka S, Fujita A, Imai H, Kogure Y, Oyama T, Asao T and Shirabe K: Prognostic significance of tumor immunity in surgically resected pulmonary pleomorphic carcinoma. Anticancer Res 40(1): 261-269, 2020. PMID: 31892575. DOI: 10.21873/anticanres.13948

16 Kodama K, Maniwa T, Kimura Y, Yagi Y, Yamamoto Y, Ohkawa T, Takeda M, Kishima H, Koh $\mathrm{G}$ and Toyofuku T: Concurrent 
combination of irradiation and immune checkpoint inhibitor for recurrent pleomorphic carcinoma of the lung. Int Cancer Conf J 7(2): 43-47, 2017. PMID: 31149513. DOI: 10.1007/s13691-017-0315-9

17 Gadgeel SM, Stevenson JP, Langer CJ, Gandhi L, Borghaei H, Patnaik A, Villaruz LC, Gubens M, Hauke R, Yang JC, Sequist LV, Bachman R, Saraf S, Raftopoulos $H$ and Papadimitrakopoulou V: Pembrolizumab and platinum-based chemotherapy as first-line therapy for advanced non-small-cell lung cancer: Phase 1 cohorts from the KEYNOTE-021 study. Lung Cancer 125: 273-281, 2018. PMID: 30429032. DOI: 10.1016/j.lungcan.2018.08.019

18 Takeda K, Matsushita H, Kubozono M, Tanabe T, Ishikawa Y, Yamamoto T, Kozumi M, Takahashi N, Katagiri Y, Tasaka S, Fukui K, Takeda K, Fujishima F, Ichinose $M$ and Jingu K: Definitive chemoradiotherapy for advanced pulmonary sarcomatoid carcinoma. Intern Med 55(22): 3325-3330, 2016 PMID: 27853077. DOI: 10.2169/internalmedicine.55.6990
19 Kato D, Chihara Y, Shirase T, Takahashi T, Takahashi KI and Sakai N: Successful treatment of two consecutive cases of pulmonary pleomorphic carcinoma with platinum chemotherapy. Oncol Lett 10(5): 3040-3042, 2015. PMID: 26722286. DOI: 10.3892/ol.2015.3678

20 Oda T, Sekine A, Kato T, Baba T, Okudela K and Ogura T: Promising effect of chemotherapy with bevacizumab for patients with pulmonary pleomorphic carcinoma: Two case reports and a literature review. Respir Investig 53(6): 296-299, 2015. PMID: 26521107. DOI: 10.1016/j.resinv.2015.05.004

Received January 21, 2020

Revised January 30, 2020

Accepted February 3, 2020 